\title{
Is administrating branched-chain amino acid-enriched nutrition achieved symptom-free in malnourished cirrhotic patients?
}

\author{
Yasuhiro Tsuda,* Hideo Fukui, Tetsuya Sujishi, Hideko Ohama, Yusuke Tsuchimoto, Akira Asai, Shinya Fukunisi \\ and Kazuhide Higuchi
}

The 2nd Department of Internal Medicine, Osaka Medical College, 2-7 Daigakumachi, Takatsuki, Osaka 569-8686, Japan

(Received 30 July, 2013; Accepted 16 August, 2013; Published online 12 December, 2013)

\begin{abstract}
Administration of branched-chain amino acids (BCAA) has been reported to improve liver function, quality of life (QOL). However, in some malnourished patients, serum albumin levels do not improve in response to BCAA granules. In this study, we examined the effects of BCAA-enriched enteral nutrition in patients unresponsive to BCAA granules. Thirty-two decompensated cirrhotic patients at Osaka Medical College were enrolled in this study. Since all patients showed no improvement in serum albumin levels despite 3 months of BCAA granule administration, they were administered $50 \mathrm{~g}$ of a flavored BCAA-enriched enteral nutrient twice daily, i.e., during the daytime and late evening. Serum albumin levels and major cirrhotic symptoms were examined 1,3 , and 5 months after treatment initiation. Serum albumin levels improved significantly $\mathbf{3}$ months after treatment initiation $(3.14 \pm 0.32 \mathrm{~g} / \mathrm{dl}$ vs $3.5 \pm 0.31 \mathrm{~g} / \mathrm{dl}, p<0.01)$, and Child-Pugh scores decreased significantly $(p<0.01)$. In the majority $(53-80 \%)$ of patients, muscles cramps, fatigue, fatigability, edema, and sleep disturbance improved within 3 months after therapy initiation. Moreover, approximately $90 \%$ of the patients became symptomfree 5 months after treatment initiation. These results indicate that switching to BCAA-enriched nutrients improves QOL of cirrhotic patients unresponsive to BCAA granules.
\end{abstract}

Key Words: BCAA-enriched nutrients, cirrhotic symptoms, QOL, LES, liver cirrhosis

D atients with liver cirrhosis (LC) suffer from malnutrition and a reduced quality of life (QOL). ${ }^{(1,2)}$ Therefore, nutritional support plays an important role in the management of LC patients. Branched-chain amino acid (BCAA) granules are commonly administered to LC patients. ${ }^{(3,4)}$ Nompleggi et al. ${ }^{(5)}$ reported that administration of BCAA granules was useful for improving serum albumin levels and Fischer's ratio in decompensated LC patients.

On the other hand, there are many cases in which serum albumin levels do not improve despite administration of BCAA granules. BCAAs play important roles in albumin synthesis and as an energy source in cases of energy deficiency. ${ }^{(6)}$ In BCAA granules ineffective patients, the majority of administrated BCAA may be used for energy. Okuda et al. ${ }^{(7)}$ reported that the administration of BCAA-enriched nutrient mixtures was useful in patients with insufficient response to BCAA granules. Furthermore, improving QOL is also a therapeutic goal for such patients. Nakaya et al $^{(8)}$ reported that once daily administration of BCAAenriched nutrient mixtures improved fatigability. Thus, administration of BCAA-enriched nutrient mixtures to LC patients with hypoalbuminemia may contribute to reduction in symptoms and improved QOL.

It has been recently shown that consumption of BCAA-enriched nutrients during a late-evening snack (LES) enhanced protein synthesis and improved nitrogen balance. ${ }^{(9,10)}$ LES has been recommended for avoiding nocturnal starvation by both the American Society for Parenteral and Enteral Nutrition (ASPEN) and the European Society for Clinical Nutrition and Metabolism (ESPEN). ${ }^{(11,12)}$

In the present study, we investigated whether twice daily administration of a BCAA-enriched nutrient mixture during LES was effective in patients with insufficient improvement in serum albumin levels or cirrhotic symptoms despite the administration of BCAA granules.

\section{Materials and Methods}

Subjects. Thirty-two decompensated cirrhotic patients at Osaka Medical College, Osaka, Japan, between December 2009 and June 2011 were enrolled in this study. Selection criteria were as follows: (i) age 20-90 years; (ii) serum albumin levels showing no improvement despite sufficient administration of BCAA granules for 3 months; and (iii) a serum albumin level $<3.5 \mathrm{~g} / \mathrm{dl}$. Exclusion criteria were as follows: (i) a serum albumin level $<2.5 \mathrm{~g} / \mathrm{dl}$ or serum bilirubin level $>3.0 \mathrm{mg} / \mathrm{dl}$; (ii) current administration of a BCAA-containing enteral nutrition product; and (iii) complications from severe diabetes mellitus. Patients' demographic data are shown in Table 1 . The daily dietary intake of each

Table 1. Demographic data

\begin{tabular}{lc}
\hline Characteristics & Values \\
\hline Age (mean \pm SD) & $72 \pm 8$ \\
Gender (M/F) & $16 / 16$ \\
Child-Pugh score & $6.8 \pm 1.2$ \\
Liver cirrhosis $(n)$ & \\
HCV & 24 \\
HBV & 3 \\
nonBnonC & 3 \\
AlH & 2 \\
AlH with PBC & 1 \\
HCC $(n)$ & 21 \\
Albumin (g/d) & $3.1 \pm 0.3$ \\
Tb (mg/dl) & $1.5 \pm 2.6$ \\
PLT $\left(\times 10^{4} \mu l\right)$ & $10.2 \pm 6.6$ \\
PT time $(\%)$ & $78.9 \pm 13.5$
\end{tabular}

Values presented by mean \pm SD HCV, hepatitis virus $C$; HBV, hepatitis virus $B$; nonBnonC, negative for hepatitis virus $B$ and $C ; A l H$, autoimmune hepatitis; PBC, primary biliary cirrhosis; HCC, hepatocellular carcinoma; Tb, total bilirubin; PLT, platelet count; PT time, prothrombin time.

*To whom correspondence should be addressed.

E-mail: in1258@poh.osaka-med.ac.jp 
patient was calculated by a dietitian and maintained at $25-35 \mathrm{kcal} /$ $\mathrm{kg}$ per day of total energy and $1.0-1.2 \mathrm{~g} / \mathrm{kg}$ per day of protein according to the ESPEN guidelines. Written informed consent was obtained from all participants.

Study design. The study was conducted according to an open-label single-arm design. Patients whose serum albumin levels showed no improvement despite sufficient administration of BCAA granules for 3 months were switched to the BCAAenriched nutrient Aminoleban EN (Otsuka Pharmaceutical Co. Ltd., Tokyo, Japan). The BCAA-enriched nutrient (50 g) was administered twice per day, including LES, for 5 months. Clinical laboratory tests including serum albumin were conducted at 0,1 , 3 , and 5 months. An original questionnaire was administered monthly during the study period to evaluate the clinical symptoms of each patient.

Evaluation. The primary endpoint was improvement in the serum albumin level and the secondary endpoint was improvement in clinical symptoms by evaluating the symptom-free ratios using a 6 -item questionnaire at each time point. The symptoms evaluated were the presence or absence of muscle cramps, fatigue, fatigability, edema, ascites, and sleep disturbance. In addition, changes in the Child-Pugh (CP) score were compared for each patient.

Statistical analysis. Improvement in serum albumin levels after BCAA-enriched nutrient treatment was analyzed by the paired $t$-test. Changes in the symptom-free ratios for each questionnaire item were analyzed by the chi-square test or Fisher's exact test. Values were expressed as differences in the symptom-free ratios at a $95 \%$ confidence interval $(\mathrm{CI})$. Statistical significance was defined as $p<0.05$. All statistical analyses were performed using JMP version 8.0.2 software (SAS Institute, Cary, NC).

\section{Results}

Clinical characteristics of patients. The characteristics of the 32 patients who met the inclusion criteria and were enrolled in this study are shown in Table 1. Among these patients, 21 had hepatocellular carcinoma. The mean baseline CP score was 6.8. BCAA-enriched enteral nutrients were administered to all the patients, except for 1 who had severe itching.

Outcomes. Serum albumin levels were significantly increased 2 months after switching to BCAA-enriched nutrients, and the mean serum albumin level reached $3.5 \mathrm{~g} / \mathrm{dl} 3$ months after initiating treatment (Fig. 1). Prothrombin times and CP scores were also significantly improved 3 months after initiation of

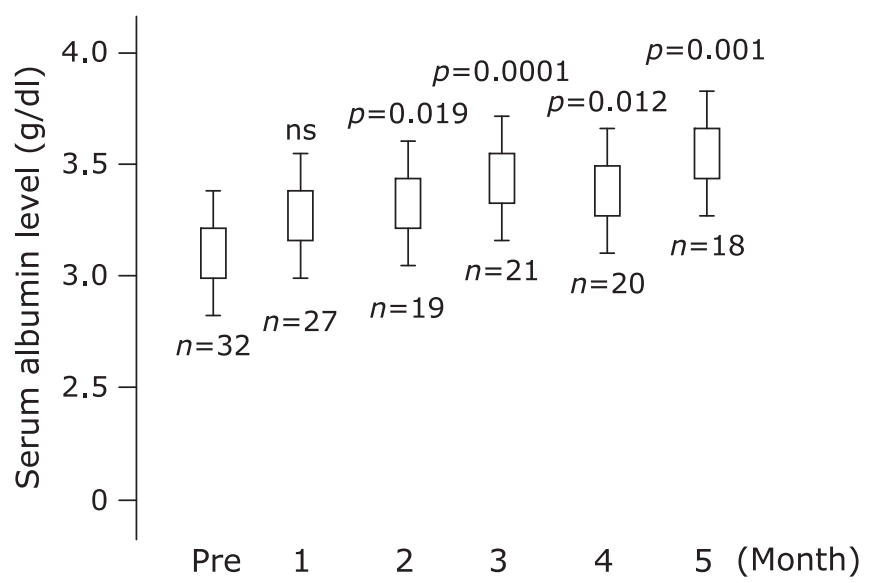

Fig. 1. Changes in serum albumin levels after switching to BCAAenriched nutrients in cirrhosis patients for whom BCAA granules were ineffective. Statistical analyses were performed by the paired $t$ test. Statistical significance was defined as $p<0.05$. ns: not significant.
Table 2. Changes in clinical laboratory tests by administrating aminoleban EN for 3 months in liver cirrhosis patients

\begin{tabular}{llcr}
\hline Clinical laboratory tests & \multicolumn{1}{c}{ Pre } & Post & $p$ value \\
\hline ALB $(\mathrm{g} / \mathrm{dl})$ & $3.18 \pm 0.33$ & $3.51 \pm 0.31$ & $<0.001$ \\
T.Bil $(\mathrm{mg} / \mathrm{dl})$ & $0.92 \pm 0.52$ & $0.92 \pm 0.55$ & 0.951 \\
PT $(\%)$ & $82.5 \pm 9.9$ & $87.8 \pm 12.5$ & 0.017 \\
Child-Pugh & $6.94 \pm 0.94$ & $6.00 \pm 0.84$ & 0.001
\end{tabular}

Values presented by mean \pm SD. ALB, albumin; T.Bil, total bilirubin; PT, prothrombin time. Statistical analysis was performed by paired $t$ test.

administration of the BCAA-enriched nutrient. Prothrombin time increased from $82.5 \% \pm 9.9 \%$ to $87.8 \% \pm 12.5 \% \quad(p=0.017$ Table 2) and CP scores improved from $6.94 \pm 0.94$ to $6.00 \pm 0.84$ ( $p=0.001$; Table 2 ) over the 3-month period.

Evaluation of symptoms. Before switching to the BCAAenriched nutrient, only $18.8 \%$ of the patients had no cirrhotic symptoms. One month after switching, cirrhotic symptoms improved in $62.5 \%$ of the patients (Fig. 2). Symptom-free ratios increased significantly each month, and 3 months after treatment initiation, up to $90 \%$ of the patients had no cirrhotic symptoms (Fig. 2). Changes in the prevalence ratios for the 6 cirrhotic symptoms from baseline to 5 months were $53.1 \%$ to $12.5 \%$ for muscle cramps, $62.5 \%$ to $21.9 \%$ for fatigue, $59.4 \%$ to $18.8 \%$ for fatigability, $37.5 \%$ to $12.5 \%$ for sleep disturbance, $15.6 \%$ to $3.1 \%$ for ascites, and $31.3 \%$ to $9.4 \%$ for edema (Table 3 ).

\section{Discussion}

In this study, we clarified that BCAA-enriched nutrient mixtures with LES were effective for improving serum albumin levels in patients for whom BCAA granules were ineffective. Moreover, in approximately $90 \%$ of these patients, 6 cirrhotic symptoms, including muscle cramps, fatigue, fatigability, sleep disturbance, ascites, and edema, had disappeared 3 months after switching to a BCAA-enriched nutrient mixture. These results indicate that switching from BCAA granule monotherapy to a BCAA-enriched nutrient mixture is effective for improving QOL of cirrhotic patients for whom BCAA granules are ineffective.

Okuda et al..$^{(7)}$ previously reported that the administration of BCAA-enriched nutrient mixtures was useful for patients with inadequate response to BCAA granules. According to their report, serum albumin levels significantly increased within 3 months after switching to a BCAA-enriched nutrient mixture. However, they did not examine changes in cirrhotic symptoms. On the other hand, the consumption of BCAA-enriched nutrients as LES has been shown to enhance protein synthesis and improve nitrogen balance. ${ }^{(9,10)}$ Therefore, we used the LES method when switching to the BCAA-enriched nutrient mixture.

Sako et al. ${ }^{(13)}$ demonstrated that administration of BCAAenriched nutrient mixtures improved muscle cramps. Previous reports also revealed that the administration of BCAA after resection or ablation of hepatocellular carcinoma improved cirrhotic symptoms in LC patients. ${ }^{(14-17)}$ However, there have been no reports of cirrhotic symptom improvement after switching to BCAA-enriched nutrients in patients for whom BCAA granules were ineffective. Our results showed improvement in 6 symptomatic items after switching to a BCAA-enriched nutrient mixture. Improvement in ascites and edema might have resulted from the elevated serum albumin levels, and improvement in fatigue, fatigability, and sleep disturbance may have resulted from improved fluid expansion status.

The BCAA-enriched nutrient mixture used in this study included $12 \mathrm{~g}$ of BCAAs, which was equivalent to the amount in BCAA granules. BCAA granules are used to supply energy in malnourished patients, such as those with cirrhosis. The BCAAenriched nutrient mixture used in this study also contains glucose, 


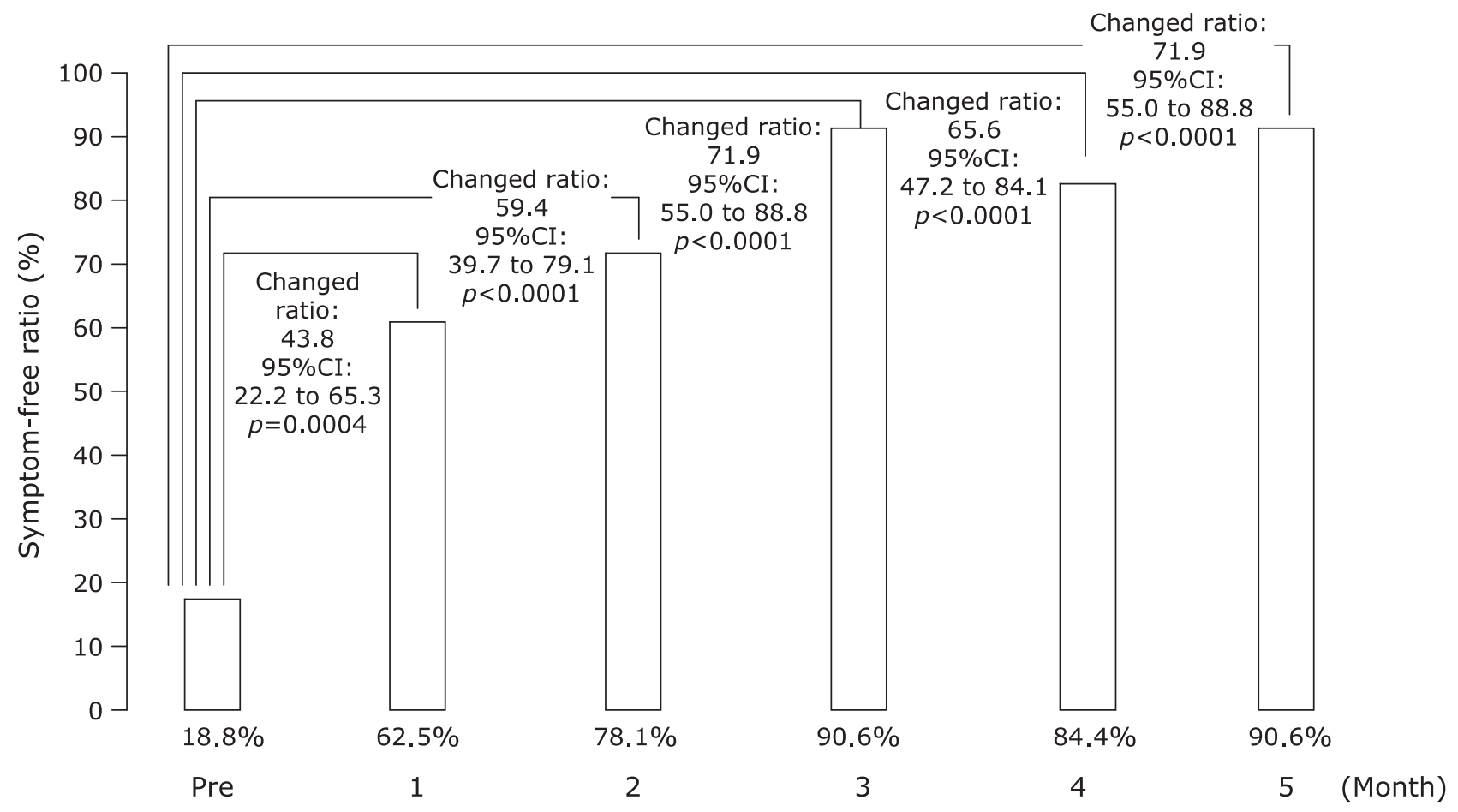

Fig. 2. Changes in symptom-free ratios after switching to BCAA-enriched nutrients in cirrhosis patients for whom BCAA granules were ineffective. Statistical analyses were performed by the paired $t$ test. Statistical significance was defined as $p<0.05$.

Table 3. Changes in symptom-prevalence ratio by administrating aminoleban EN in hepatic cirrhosis patients

\begin{tabular}{lcrrrrc}
\hline Symptom & Pre & 1 & 2 & 3 & 4 & 5 (month) \\
\hline Muscle cramp & $53.1 \%$ & $34.4 \%$ & $25.0 \%$ & $15.6 \%$ & $15.6 \%$ & $12.5 \%$ \\
Fatigue & $62.5 \%$ & $34.4 \%$ & $21.9 \%$ & $18.8 \%$ & $21.9 \%$ & $21.9 \%$ \\
Fatigability & $59.4 \%$ & $40.6 \%$ & $21.9 \%$ & $18.8 \%$ & $18.8 \%$ & $18.8 \%$ \\
Sleep disturbance & $37.5 \%$ & $28.1 \%$ & $15.6 \%$ & $15.6 \%$ & $15.6 \%$ & $12.5 \%$ \\
Ascites & $15.6 \%$ & $6.3 \%$ & $6.3 \%$ & $3.1 \%$ & $3.1 \%$ & $3.1 \%$ \\
Edema & $31.3 \%$ & $9.4 \%$ & $6.3 \%$ & $6.3 \%$ & $9.4 \%$ & $9.4 \%$ \\
\hline
\end{tabular}

lipids, and various other nutrients, which may have contributed to improved protein synthesis. In addition, as reported previously, LES administration may contribute to improvement in albumin synthesis. ${ }^{(9,10,14)}$ The minerals and/or vitamins included in the BCAA-enriched nutrient mixture may play a role in improvement of cirrhotic symptoms.

In this study, fatigue, fatigability, and sleep disturbance improved in approximately $70 \%$ of patients for whom BCAA granules were ineffective, suggesting that the BCAA-enriched nutrient was effective for improving QOL in these LC patients. The prognosis is poor for severely decompensated LC patients; ${ }^{(17,18)}$ therefore, improved QOL could be an important terminal therapeutic strategy. Evaluation of QOL is difficult because of the various psychological factors involved. The SF-8 Health Survey and the Functional Assessment of Cancer Therapy have been used in some previous studies. ${ }^{(16,17)}$ In this study, we used an original questionnaire to focus on 6 symptomatic items. Although there are several methods for demonstrating improvement in QOL, no

\section{References}

1 Lautz HU, Selberg O, Körber J, Bürger J, Müller MJ. Protein-calorie malnutrition in liver cirrhosis. Clin Investig 1992; 70: 478-486.

2 Italian Multicentre Cooperative Project on nutrition in liver cirrhosis. Nutritional status in cirrhosis. Nutritional status in cirrhosis. J Hepatol 1994; method evaluates the elimination of each symptom. Tally et al. ${ }^{(19)}$ demonstrated that the most important question was "Will you continue to take the study drug when finished with this trial?". This question is important when symptoms are being evaluated, and we intend to include it in future questionnaires.

In conclusion, the twice-daily administration of a BCAAenriched nutrient mixture during LES contributed to improvement in cirrhotic symptoms in LC patients with inadequate response to BCAA granules. Thus, decompensated cirrhotic patients with insufficient elevation of serum albumin despite optimal BCAA granule administration may require similar nutritional adjustments.

\section{Conflict of Interest}

Kazuhide Higuchi received a research grant from Otsuka Pharmaceutical Inc. 
4 Miwa Y, Fukushima H, Moriwaki H, Muto Y. Nutritional pharmacotherapy of liver cirrhosis for prognosis and improvement of quality of life. J Clin Biochem Nutr 2002; 31: 1-8.

5 Nompleggi DJ, Bonkovsky HL. Nutritional supplementation in chronic liver disease: an analytical review. Hepatology 1994; 19: 518-533.

6 Tessari P, Zanetti M, Barazzoni R, et al. Response of phenylalanine and leucine kinetics to branched chain-enriched amino acids and insulin in patients with cirrhosis. Gastroenterology 1996; 111: 127-137.

7 Okuda H, Hashimoto E, Hasegawa K, Hayashi N. Nutritional evaluation of BCAA supplements (AminolebanEN) in cirrhotic patients. JJPEN 2002; 24: 553-556.

8 Nakaya Y, Okita K, Suzuki K, et al. BCAA-enriched snack improves nutritional state of cirrhosis. Nutrition 2007; 23: 113-120.

9 Fukushima H, Miwa Y, Ida E, et al. Nocturnal branched-chain amino acid administration improves protein metabolism in patients with liver cirrhosis: comparison with daytime administration. JPEN J Parenter Enteral Nutr 2003; 27: 315-322.

10 Tsuchiya M, Sakaida I, Okamoto M, Okita K. The effect of a late evening snack in patients with liver cirrhosis. Hepatol Res 2005; 31: 95-103.

11 ASPEN Board of Directors and the Clinical Guidelines Task Force. Guidelines for the use of parenteral and enteral nutrition in adult and pediatric patients. JPEN J Parenter Enteral Nutr 2002; 26: 1SA-138SA.

12 Plauth M, Merli M, Kondrup J, et al. ESPEN guidelines for nutrition in liver disease and transplantation. Clin Nutr 1997; 16: 43-55.
13 Sako K, Imamura Y, Nishimata H, Tahara K, Kubozono O, Tsubouchi $\mathrm{H}$. Branched-chain amino acids supplements in the late evening decrease the frequency of muscle cramps with advanced hepatic cirrhosis. Hepatol Res 2003; 26: 327-329.

14 Morihara D, Iwata K, Hanano T, et al. Late-evening snack with branchedchain amino acids improves liver function after radiofrequency ablation for hepatocellular carcinoma. Hepatology Res 2012; 42: 658-667.

15 Okabayashi T, Nishimori I, Sugimoto T, et al. Effects of branched-chain amino acids-enriched nutrient support for patients undergoing liver resection for hepatocellular carcinoma. J Gastroenterol Hepatol 2008; 23: 1869-1873.

16 Kuroda H, Ushio A, Miyamoto Y, et al. Effects of branched-chain amino acid-enriched nutrient for patients with hepatocellular carcinoma following radiofrequency ablation: a one-year prospective trial. J Gastroenterol Hepatol 2010; 25: 1550-1555.

17 Poon RT, Yu WC, Fan ST, Wong J. Long-term oral branched chain amino acids in patients undergoing chemoembolization for hepatocellular carcinoma: a randomized trial. Aliment Pharmacol Ther 2004; 19: 779-788.

18 Boursier J, Cesbron E, Tropet AL, Pilette C. Comparison and improvement of MELD and Child-Pugh score accuracies for the prediction of 6-month mortality in cirrhotic patients. J Clin Gastroenterol 2009; 43: 580-585.

19 Talley NJ, Riff DS, Schwartz H, Marcuard SP. Double-blind placebocontrolled multicentre studies of rebamipide, a gastroprotective drug, in the treatment of functional dyspepsia with or without Helicobacter pylori infection. Aliment Pharmacol Ther 2001; 15: 1603-1611. 